\title{
ESTUDO COMPARATIVO DO POTENCIAL INFLAMATÓRIO DE EXTRATOS VEGETAIS INSETICIDAS
}

\section{COMPARATIVE STUDY OF INFLAMMATORY POTENTIAL OF INSECTICIDES VEGETABLES EXTRACTS}

Mariana W. Andrade Barcella', Norma Catarina Bueno², Edson A. Alves da Silva ${ }^{3}$, Rafael Andrade Menolli ${ }^{4}$, Carla Brugin Marek ${ }^{4}$, Tereza Cristina M. Jorge ${ }^{4^{*}}$.

'Acadêmica de Farmácia da Universidade Estadual do Oeste do Paraná UNIOESTE; ${ }^{2}$ Professora de Biologia do Centro de Ciências Biológicas e da Saúde, UNIOESTE; ${ }^{3}$ Professor de Estatística do Centro de Ciências Exatas e Tecnológicas, UNIOESTE, ${ }^{4}$ Professores do Centro de Ciências Médicas e Farmacêuticas, UNIOESTE.

"E-mail: tcmjorge@unioeste.br

\section{RESUMO:}

Pediculose é uma infestação causada pelo parasita Pediculus humanus capitis De Geer, conhecido popularmente como piolho. A doença é considerada um problema sério de saúde pública. Embora existam diversos tratamentos populares para a infestação, poucos estudos científicos comprovam a eficácia dos tratamentos. A utilização de espécies vegetais com atividade inseticida antipediculose, além de acessível à população de baixa renda, também se constitui em tratamento alternativo eficaz para o controle da doença. Os extratos vegetais obtidos de Melia azedarach L., Ruta graveolens L. e Sambucus australis Cham. et Schlecht apresentam atividade antipediculose, no entanto é importante investigar o potencial inflamatório destes extratos. O presente trabalho analisou o poder de indução inflamatória de extratos hidroalcoólicos das espécies vegetais $M$. azedarach, $R$. graveolens e Sambucus australis, empregando ratos da linhagem Wistar. Neste estudo não foi possível constatar a existência de inflamação significativa produzida pelos três extratos vegetais nos níveis dos tempos investigados.

Palavras-chaves: Melia azedarach, Ruta graveolens, Sambucus australis, inflamação.

\section{ABSTRACT:}

Pediculosis is an infestation caused by the parasite Pediculus humanus capitis de Geer, popularly known as lice. The disease is considered a serious public health problem. Although there are several popular treatments for the infestation, few scientific studies have proven their efficacy. The use of vegetal species with anti pediculosis insecticide activity, besides being accessible to low income population, also constitutes an efficient 
alternative treatment to control the disease. The vegetal extracts obtained from Melia azerach L, Ruta graveolens L e Sambucus australis Cham. Et Schelecht have shown anti pediculosis activity. However, it is important to investigate the inflammatory potential of this kind of extract. This work has analyzed the power to induce inflammation of the hidroalcoholic vegetais species $M$. azerach, $R$. graveolens e Sambucus australis in Wistar rats. In this study, it was not possible to observe the existence of significant inflammation produced by the three extracts in the levels of the times investigated.

Keywords: Melia azedarach, Ruta graveolens, Sambucus australis, inflammation.

\section{INTRODUÇÃO}

As espécies vegetais: Melia azedarach L., conhecida como erva de santabárbara ou cinamomo (Fig.1a), Ruta graveolens L. ou arruda (Fig.1b), e Sambucus australis Cham. et Schlecht ou sabugueiro (Fig.1c) são frequentemente utilizadas em medicina popular, porque apresentam atividades biológicas importantes.
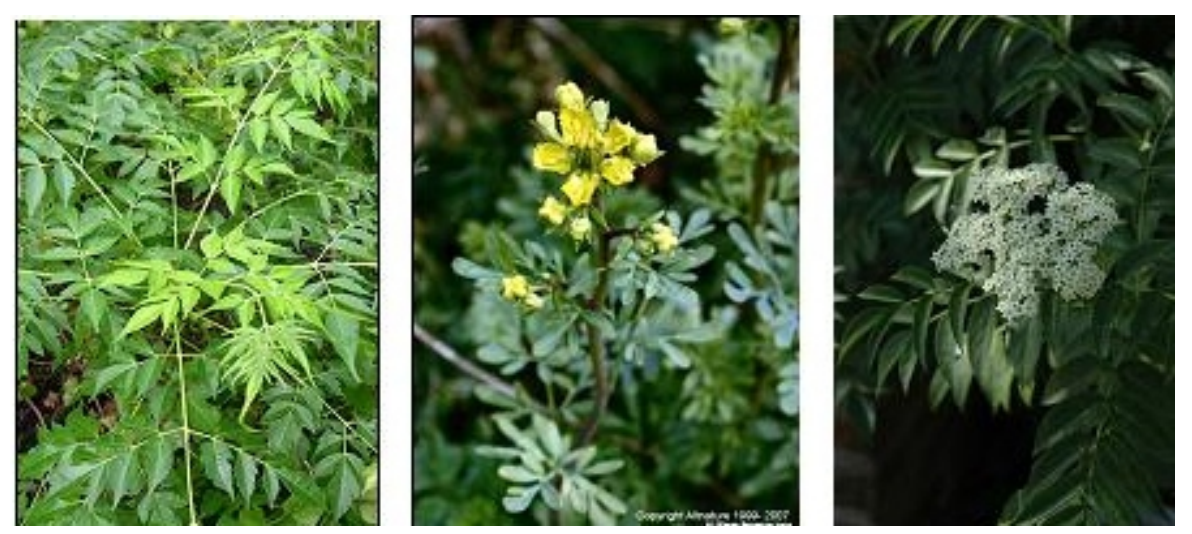

FIGURA 1: (a) Melia azedarach, (b) Ruta graveolense (c) Sambucus australis Fonte disponível em:

(a) http://www.nps.gov/plants/alien/fact/meaz1.htm,

(b) http://www.herbies-herbs.com/pages/herbpictures/rue2504L.jpg e

(c) http://www.viveroianni.com.ar/\%5Cimagenes\%5CSambucus_australis.jpg

A atividade inseticida da espécie Melia azedarach já foi estudada e há vários registros na literatura científica sobre o assunto, por exemplo: Liu (2000), Defago (2006) e Kim (2006). Derivados terpênicos são constituintes desta espécie vegetal, sendo isolados em pesquisa fitoquímica como a realizada por Viegas (2003). A propriedade larvicida desta planta também foi demonstrada por Maciel (2006) e, Nathan (2006) verificou que o extrato bruto é ativo contra o mosquito Anopheles. A picada deste inseto transmite o protozoário do gênero Plasmodium, causador da malária. Wandscheer 
(2004) também estudou o extrato bruto de $M$. azedarach e concluiu que, além do mosquito Anopheles, o extrato também é ativo contra o mosquito Aedes Aegypti, causador da dengue.

As folhas da espécie Ruta graveolens também apresentam atividade inseticida (Franco, 2001). A medicina popular registra utilizações desta planta em tratamentos de supressão da menstruação humana e os extratos são considerados antimicrobianos, citotóxicos (Ivanova, 2005), algicidas e fungicidas (MEEPAGALA, 2005).

A espécie Sambucus australis é empregada em tratamentos de ferimentos traumáticos, sob a forma de emplastro medicinal (LAN, 2006). As folhas frescas apresentam atividade inseticida, sendo muito utilizadas em medicina popular para tratar a pediculose (CÔRREA, 1998).

A pediculose é a infestação da cabeça causada por piolho, parasita cujo nome científico é Pediculus humanus capitis De Geer (CASTELO-BRANCO, 2004). No Brasil, a infestação atinge cerca de $30 \%$ das crianças em fase escolar. Segundo Barbosa (2003) vários medicamentos antipediculose estão disponíveis no mercado, destacando-se produtos à base de organofosforados e de piretróides, como a deltametrina e permetrina, que podem apresentar efeitos colaterais.

A população brasileira carente tem dificuldade em adquirir estes produtos e utiliza tratamentos mais baratos e caseiros, à base de soluções produzidas, por exemplo, a partir de boldo (Plectranthus barbatus), melão de São Caetano (Momordica charantia) e arruda. Estes tratamentos podem ser promissores, porém pouco se sabe sobre o combate efetivo ao piolho. Os dados são empíricos e seus resultados não estão registrados na literatura científica. Estima-se que milhares de pessoas estejam infestadas por piolhos, fato que revela a importância de um tratamento alternativo e eficaz para o controle da doença.

Os resultados antipediculose obtidos por Jorge e colaboradores (2009) mostraram que o piolho é sensível a tratamentos com extratos hidroalcoólicos das folhas frescas de $S$. australis, M. azedarach e R. graveolens. No entanto, nada se conhece sobre a toxidade e potencial inflamatório destes extratos. Silva (2004) e Souza (2005) realizaram um experimento, em que estudaram o potencial inflamatório de extratos vegetais, colocando os extratos em contato com a pele de cobaias e, após algum tempo, observaram a produção de inflamação. Tomamos por base este experimento e realizamos outro trabalho semelhante. Neste trabalho utilizamos ratos da linhagem Wistar e nosso objetivo foi investigar a indução inflamatória de extratos hidroalcoólicos de $M$. azedarach, R. graveolens e S. australis em contato com a derme dessas cobaias, em diferentes tempos de exposição. 


\section{MATERIAL E MÉTODOS}

\subsection{Delineamento Estatístico}

Foi idealizado um delineamento experimental casualisado, com 3 blocos completos. Cada bloco representou um de três tempos de exposição dos extratos vegetais com a pele das cobaias (30,60 e 180 minutos). Para cada tempo de exposição utilizou-se 7 repetições. Cada rato recebeu 4 tratamentos: 3 de extratos diferentes das plantas e 1 de solução salina como testemunha (Figura 2). A análise estatística foi feita com o programa R (R Development Core Team, 2011), gratuito e de código aberto.

\subsection{Obtenção do Material Vegetal}

Folhas e galhos das três espécies vegetais foram coletados no campus universitário da Universidade Estadual do Oeste do Paraná (UNIOESTE), Cascavel e identificadas pela bióloga Prof ${ }^{\mathrm{a}} \mathrm{Dr}^{\mathrm{a}}$. Norma Catarina Bueno. O extrato de cada planta foi obtido, após filtração, pesando-se $300 \mathrm{~g}$ de folhas e galhos, submetidos à maceração por 48 horas em um litro de solução hidroalcoólica 50\%.

\subsection{Teste do Potencial Inflamatório}

Cobaias da linhagem Wistar (21 ratos), do sexo masculino, com idade de 4 meses e pesando aproximadamente $370 \mathrm{~g}$ foram cedidos pelo Biotério da UNIOESTE. Os animais foram anestesiados antes do início do experimento e a pelagem do dorso foi retirada. O corante "Azul de Evans" foi aplicado na veia caudal lateral de cada animal na concentração de $2 \%$ (20 mg. $\left.\mathrm{Kg}^{-1}\right)$. A seguir, 0,1 mL de cada extrato vegetal (tratamento) mais $0,1 \mathrm{~mL}$ de solução salina (controle) foram aplicados via intradérmica, em quatro regiões do dorso de cada animal (Figura 2).

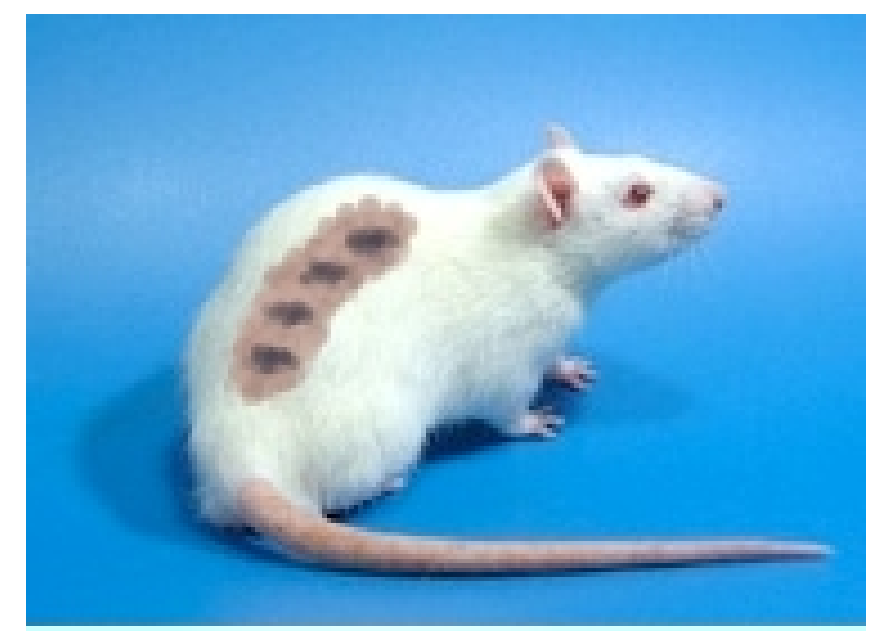

Figura 2. Esquema de aplicação dos extratos vegetais e de solução salina no dorso da cobaia. 
Fragmentos de pele, onde se aplicou cada extrato, foram dissecados, depois de transcorrido o intervalo de tempo atribuído a cada grupo. O corante "Azul de Evans" foi utilizado, porque precipita in vivo, nas regiões afetadas por processos inflamatórios. Assim, cada fragmento de pele dissecado foi imerso em tubo de ensaio, contendo $10 \mathrm{~mL}$ de solução aquosa de dimetilformamida (DMF) $50 \%$, durante 72 horas, à $45^{\circ} \mathrm{C}$. Este procedimento dissolve e extrai o corante presente na derme das cobaias, de forma que locais com processo inflamatório mais intenso, mostram coloração azul mais acentuada. Os dados para análise e comparação dos potenciais inflamatórios foram obtidos, após filtração dos conteúdos dos tubos de ensaio. Medidas de absorções de luz UV foram realizadas com os filtrados em espectrofotômetro Ultra-Violeta (UV) marca Wazelength, modelo SP 1105 (= 620 m). As absorbâncias das soluções coloridas foram medidas e relacionadas ao potencial inflamatório produzido por cada extrato vegetal.

\section{RESULTADOS E DISCUSSÃO}

A Tabela 1 apresenta as médias e desvios padrões das absorbâncias obtidas de soluções coloridas, produzidas com o corante "Azul de Evans", em tempo controlado, após aplicação de cada extrato nas cobaias.

TABELA 1. Médias e desvios padrões das absorbâncias dos extratos.

\begin{tabular}{lllllllll}
\hline \multirow{2}{*}{$\begin{array}{l}\text { Tempo } \\
\text { (minutos) }\end{array}$} & \multicolumn{9}{c}{ S. australis } & \multicolumn{3}{c}{$\boldsymbol{R}$. graveolens } & \multicolumn{2}{c}{ M. azedarach } & \multicolumn{3}{c}{ Solução salina } \\
\cline { 2 - 9 } & Média & DP & Média & DP & Média & DP & Média & DP \\
30 & 0,165 & 0,007 & 0,229 & 0,002 & 0,160 & 0,007 & 0,052 & 0,0003 \\
60 & 0,222 & 0,003 & 0,312 & 0,006 & 0,155 & 0,001 & 0,071 & 0,0007 \\
180 & 0,333 & 0,013 & 0,333 & 0,011 & 0,370 & 0,019 & 0,054 & 0,0003 \\
\hline
\end{tabular}

DP: Desvio Padrão.

O gráfico da Figura 3 foi produzido com dados da Tabela 1 e ilustra a distribuição das absorbâncias. Nota-se uma grande assimetria positiva, indicando tendência dos resultados aos menores valores e uma grande semelhança das absorbâncias médias, segundo os tratamentos. 


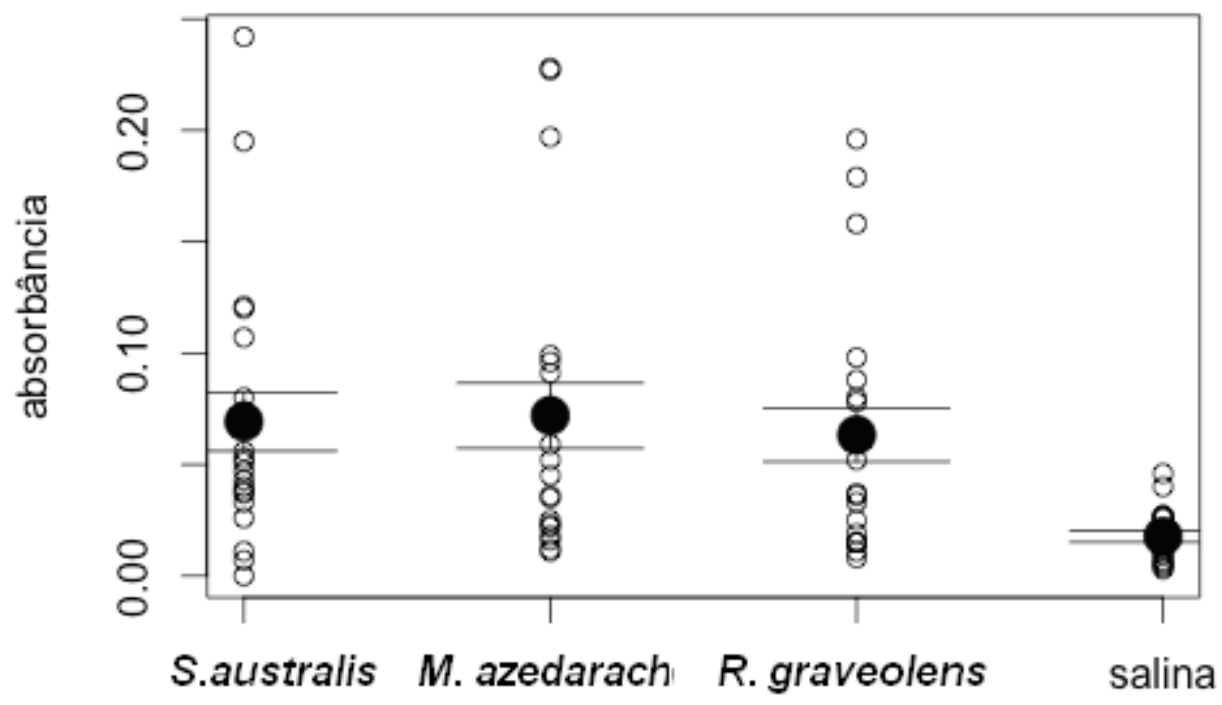

FIGURA 3: Distribuição das médias e erros padrões de absorbâncias dos extratos vegetais de S.australis, M.azedarach, R.graveolens e Salina.

Devido à natureza da distribuição dos resultados, optou-se pelo teste não paramétrico de Levene para investigar o efeito dos tratamentos. Observou-se que não houve efeito significativo, pois os resultados são comparáveis com a solução salina ( $p$ valor $=0,1218$ ), embora a Tabela 1 e a Figura 1 sugiram que os extratos de $S$. australis, $R$. graveolens e $M$. azedarach apresentem valores crescentes das absorbâncias médias nos tempo de 30, 60 e 180 minutos e as absorbâncias da solução salina (controle) não apresentem o mesmo efeito durante o tempo observado.

\section{CONCLUSÕES}

A realização deste trabalho possibilitou avaliar o potencial inflamatório que os tratamentos de extratos hidroalcoólicos de S.australis, R.graveolens e M.azedarach exercem sobre a derme de cobaias nos períodos de 30,60 e 180 minutos. Neste estudo não foi possível constatar a existência de inflamação significativa produzida pelos três extratos vegetais nos níveis de tempos investigados.

\section{AGRADECIMENTOS}

Ao Biotério da UNIOESTE pelo fornecimento das cobaias e à Fundação Araucária pela bolsa de estudo concedida. 


\section{REFERÊNCIAS}

BARBOSA, J.V; PINTO, Z.T. Pediculose no Brasil. Entomol. Vect. 2003, 10 (4), 579583.

BRANCO, C.W.B; SARTI, S.J.; CESTARI, I.M. Evoluation of the Potential Inseticide Activy of Tagets minuta Essencial Oil Against Head Lice Pediculus humanus capitis. Neotropical Entomology. 2004, 33 (6), 805-807.

CÔRREA, A.D.; SIQUEIRA, R.B.; QUINTAS, L.E.M. Plantas Medicinais - Do cultivo à terapêutica. Petrópolis: Ed. Vozes, 1998.

DEFAGO, M.; VALLADARES, G. et al. Insecticide and antifeedant activity of different plant parts of Melia azedarach on Xanthogaleruca luteola. Fitoterapia, 2006, 77 (7-8), 500-505.

FRANCO, I.J.; Fontana, V. L. Ervas e Plantas - A medicina dos simples. Erexim: Ed. Edelbra, 2001.

IVANOVA, A.; MIKHOVA, B.; NAJDENSKI, H. et al. Antimicrobial and cytotoxic activity of Ruta graveolens. Fitoterapia, 2005, 76 (3-4), 344-347.

JORGE, T.C.M., LENARTOVICZ, V.; ANDRADE, M.W.; BONAFIN, T.;

GIORDANI, M.A.; BUENO, N.C.; SCHNEIDER, D.S.L.G. Lat. Am. J. Pharm., 2009, 28, (3), 457-459.

KIM, K.S.; JANG, K.H. Natural insecticide containing extracts of plants capable of inhibiting proliferation of insects without harmful effects. Repub. Korean Kongkae Taeho Kongbo, 2006.

LAN, Z. Chinese medicinal plaster for treating traumatic injury and rheumatic bone and joint diseases. Faming Zhuanli Shenqing Gongkai Shuomingshu, 2006.

LIU, X. Insecticidal attractant. Faming Zhuanli Shenqing Gongkai Shuomingshu, 2000.

MACIEL, M.V.; MORAIS, S.M. et al. Ovicidal and larvicidal activity of Melia azedarach extracts on Haemonchus contortus. Vet. Parasitol. 2006, 140 (1-2), 98-104.

MEEPAGALA, K.A.; SCHRADER, K.K.; WEDGE, D.E. et al. Algicidal and antifungal 
compounds from the roots of Ruta graveolens and synthesis of their analogs. Phytochemistry, 2005, 66 (22), 2689-2695.

NATHAN, S.S.; SAVITHA, G.; GEORGE, D.K. et al. Efficacy of Melia azedarach L. extract on the malarial vector Anopheles stephensi Liston (Diptera: Culicidae). Bioresource Technol., 2006, 97 (11), 1316-1323.

R Development Core Team. R: A language and environment for statistical computing. $R$ Foundation for Statistical Computing, Vienna, Austria, 2011. Disponível em: http://www.R-project.org/.> Acesso em: 09.03.2012.

SILVA, F.B.; ALMEIDA, J.M.; SOUSA, S.M.G. Natural medicaments in endodontics - a comparative study of the anti-inflammatory action. Braz Oral Res., 2004, 18, (2) 174-9. SOUSA, S.M.G.; BRAMANTE, C.M.; TAGA, E.M. Biocompartibility of EDTA, EGTA and citric acid. Braz Oral Res., 2005, 16, (1), 3-8.

VIEGAS, C. Terpenes with insecticidal activity: an alternative to chemical control of insects. Quim Nova, 2003, 26 (3), 390-400.

WANDSCHEER, C.B.; DUQUE, J.E. et al. Larvicidal action of ethanolic extracts from fruit endocarps of Melia azedarach and Azadirachta indica against the dengue mosquito Aedes aegypti. Toxicon., 2004, 44 (8), 829-835. 